\title{
A Toolbox for Analyzing and Testing Mode Identification Techniques and Network Equivalent Models
}

\author{
Eleftherios O. Kontis ${ }^{1}$, Georgios A. Barzegkar-Ntovom ${ }^{2}{ }^{(D}$, Konstantinos A. Staios ${ }^{1}$, \\ Theofilos A. Papadopoulos ${ }^{2}$ D and Grigoris K. Papagiannis ${ }^{1, *}$ \\ 1 Power Systems Laboratory, School of Electrical and Computer Engineering, \\ Aristotle University of Thessaloniki, GR-54124 Thessaloniki, Greece \\ 2 Power Systems Laboratory, Department of Electrical and Computer Engineering, \\ Democritus University of Thrace, GR-67100 Xanthi, Greece \\ * Correspondence: grigoris@eng.auth.gr; Tel.: +30-2310-9963-88
}

Received: 14 June 2019; Accepted: 4 July 2019; Published: 6 July 2019 updates

\begin{abstract}
During the last decade the dynamic properties of power systems have been altered drastically, due to the emerge of new non-conventional types of loads as well as to the increasing penetration of distributed generation. To analyze the power system dynamics and develop accurate models, measurement-based techniques are usually employed by academia and power system operators. In this regard, in this paper an identification toolbox is developed for the derivation of measurement-based equivalent models and the analysis of dynamic responses. The toolbox incorporates eight of the most widely used mode identification techniques as well as several static and dynamic network equivalencing models. First, the theoretical background of the mode identification techniques as well as the mathematical formulation of the examined equivalent models is presented and analyzed. Additionally, multi-signal analysis methods are incorporated in the toolbox to facilitate the development of robust equivalent models. Additionally, an iterative procedure is adopted to automatically determine the optimal order of the derived models. The capabilities of the toolbox are demonstrated using simulation responses, acquired from large-scale benchmark power systems, as well as using measurements recorded at a laboratory-scale active distribution network.
\end{abstract}

Keywords: equivalent models; graphical user interface; load modelling; mode identification; multi-signal analysis; power system dynamics

\section{Introduction}

\subsection{Motivation, Literature Review and Scope of the Paper}

Traditionally, the dynamic analysis of transmission and distribution grids is performed by using detailed power system models [1,2]. However, with the increased penetration of distributed renewable energy sources into the existing power systems [3], the use of new types of loads, e.g., power electronic loads, the need for more operational flexibility and the application of advanced voltage and frequency control strategies at the distribution level, it is becoming more and more difficult for operators and utilities to develop and maintain detailed and accurate power system models $[4,5]$. To overcome these issues, the application of measurement-based methods has been proposed as a supplementary or even principal solution for the dynamic analysis of modern power systems. In this context, several measurement-based mode identification techniques [1] and network equivalent models [6] have been proposed in the literature. 
Measurement-based mode identification techniques use dynamic responses, acquired either from ringdown or operational ambient data [1], to estimate the oscillatory modes of the grid and to predict the stability margins. Based on the form of the data they use, measurement-based mode identification techniques can be classified into time-domain (TD) and frequency domain (FD) [7]. The former apply directly to the measured data [7], while in the latter the signal needs first to be transformed to the FD in order to extract the contained mode parameters [7]. Measurement-based mode identification methods are mainly used for real-time monitoring of inter-area oscillations [8], transient stability assessment [9], wide-area control applications [10], and for the fine tuning of power system stabilizers [11].

In situ measurements can also be used for the development of reduced order equivalent models [6]. Network models can be used to facilitate the analysis of complex power systems by replacing specific parts of the examined grid [2]. They are divided into two main categories, i.e., static and dynamic equivalent models [6]. Static equivalents express the real and reactive power of the grid at any time instant as algebraic functions of the grid voltage magnitude and/or frequency at that specific time instant [6]. On the other hand, dynamic equivalents express the real and reactive power at any time instant as function of time, voltage and/or frequency [6]. Dynamic equivalents generally use difference or differential equations [6]. Static equivalents are mostly used for power flow analysis [6], while dynamic equivalents are used for small signal and transient stability analysis [12], frequency stability analysis [13], and long- and short-term voltage stability studies [14].

Scope of this paper is to facilitate and promote the use of measurement-based techniques for the analysis of modern power systems, by developing the System Identification and Equivalent Models Toolbox (SIEMToolbox). SIEMToolbox is suitable for the analysis and testing of mode identification techniques and power system equivalent models. The toolbox is developed in the MATLAB programming language and is supported by a complete graphical user interface (GUI) and a comprehensive user manual. SIEMToolbox is available for download at [15].

\subsection{Functionalities of System Identification and Equivalent Models Toolbox (SIEMToolbox)}

SIEMToolbox readily supports six of the most well-known and well-established mode identification techniques [1], namely the Prony method [16], the matrix pencil (MP) technique [17], the eigenvalue realization algorithm (ERA) [18], the prediction error method (PEM) [19], the sub-space state-space system identification (N4SID) method [20], and the fast Fourier transform (FFT) combined with the sliding window technique [21]. The first five methods apply to the TD, while FFT operates on the FD. Additionally, SIEMToolbox supports the use of the Vector Fitting (VF) algorithm [22], which operates on the FD for the identification of power system modes [23], as well as a hybrid FD/TD method [24], which has been proved very robust and accurate for the mode identification of active distribution networks (ADNs) [25]. Moreover, SIEMToolbox is equipped with an algorithmic procedure aiming to identify automatically the dominant modes contained in power system responses using data acquired either from individual or multiple signals. Thus, SIEMToolbox can ensure the development of minimum order models.

Additionally, the developed toolbox supports several network equivalent models developed during the last decades. Most of them are included in the reports of relative IEEE Task Forces [26,27] as well as in the recent report "Modelling and aggregation of loads in flexible power networks" of the International Council on Large Electric Systems (CIGRE) working group C4.605 [28]. Specifically, seven types of static equivalent models and two types of dynamic equivalent models are incorporated into the toolbox. Static equivalents include the well-known Electric Power Research Institute (EPRI) model [29], the exponential (EXP) model [26], the polynomial or ZIP model [26], the ZIP-exponential model [30] as well as the static equivalents proposed by Sabir in [31], Ohyama in [32], and Frantz in [33]. Note that EPRI, EXP, ZIP and ZIP-EXP models are developed both with and without considering a frequency-dependent term. On the other hand, dynamic equivalents include models based on transfer functions (TF) [34] as well as models based on a combination of transfer functions and non-linear equations (TFNL) [35]. The optimal order of the transfer functions and the model parameters 
are determined through an automated algorithmic procedure which supports the analysis of both individual and multiple signals.

The rest of the paper is organized as follows. In Section 2 the theoretical background of mode identification methods and network equivalencing is discussed. In Section 3 the main functionalities and capabilities of the SIEMToolbox are presented. Section 4 presents the network topologies employed to obtain the required responses. Furthermore, indicative results are presented to validate the effectiveness of the SIEMToolbox. Finally, Section 5 concludes the paper.

\section{Problem Formulation and Parameter Estimation}

In the next Sections the background of mode identification from dynamic responses and modelling of network equivalents is described. The corresponding calculation procedures implemented in the SIEMToolbox are also presented in detail.

\subsection{Mode Identifcation}

\subsubsection{Problem Formulation}

The performance of linear and time-invariant dynamic systems can be analyzed using the state-space representation of (1) and (2), assuming that the time variable $t$ is continuous $(t \in \mathbb{R})$ [1]. If the system is subjected to small perturbations, (1) and (2) are evaluated at the operating point, around which the perturbation is considered [36].

$$
\begin{gathered}
\dot{\mathbf{x}}(t)=\mathbf{A x}(t)+\mathbf{B u}(t)+\mathbf{w}(t), \\
\mathbf{y}(t)=\mathbf{C} \mathbf{x}(t)+\mathbf{D} \mathbf{u}(t)+\mathbf{v}(t)
\end{gathered}
$$

In (1) and (2) column vectors $\mathbf{y} \in R^{m \times 1}, \mathbf{u} \in R^{r \times 1}$ are the output and input system responses, respectively and $\mathbf{x} \in R^{n \times 1}$ is the state vector. $\mathbf{A} \in R^{n \times n}, \mathbf{B} \in R^{n \times r}, \mathbf{C} \in R^{m \times n}$ and $\mathbf{D} \in R^{m \times r}$ are the system matrices, whereas $\mathbf{w} \in R^{n \times 1}$ and $\mathbf{v} \in R^{m \times 1}$ are the measurement and the process noise vectors, respectively. Both noise vectors are assumed to be zero mean, stationary white noise vector sequences and uncorrelated with the system inputs. The response of each system state $x_{j}$ can be described as the sum of $n$ system modes [25]:

$$
x_{j}(t)=\sum_{i=1}^{n} c_{i} e^{\lambda_{i} t}=\sum_{i=1}^{n} a_{i} e^{\sigma_{i} t} \cos \left(\omega_{i} t+\varphi_{i}\right),
$$

where $\lambda_{i}=\sigma_{i} \pm j \omega_{i}$ are the $n$ eigenvalues of matrix $\mathbf{A}$ and can be either purely real or appear in complex conjugate pairs; $\omega_{i}=2 \pi f_{i}$ and $\sigma_{i}$ correspond to the mode angular frequency (rad/s) and damping factor $(1 / \mathrm{s})$. The parameter $c_{i}=a_{i} e^{ \pm j \varphi_{i}} / 2$ is the residue of the $i$-th mode, whereas $a_{i}$ and $\varphi_{i}$ are the amplitude and the phase angle, respectively.

The discrete form of (1) and (2) at time instant $k$ is rewritten in (4) and (5), assuming a discretization of $F_{S}=1 / T_{S}$ samples per second that generates $N$ samples [1].

$$
\begin{gathered}
\mathbf{x}_{k+1}=\mathbf{A} \mathbf{x}_{k}+\mathbf{B} \mathbf{u}_{k}+\mathbf{w}_{k} \\
\mathbf{y}_{k}=\mathbf{C} \mathbf{x}_{k}+\mathbf{D} \mathbf{u}_{k}+\mathbf{v}_{k}
\end{gathered}
$$

The discrete-time poles $\left(z_{i}\right)$ are related to $\lambda_{i}$ with:

$$
z_{i}=e^{\lambda_{i} T_{s}}
$$


If the system under consideration is single-input single-output (SISO), the transfer function representation is preferable. The transfer function of a SISO system in FD is defined in (7), assuming that $\mathbf{D}=0$ :

$$
H(s)=\frac{Y(s)}{U(s)}=\sum_{i=1}^{n} \frac{c_{i}}{s-p_{i}}
$$

where $s=j \omega$ and $p_{i}$ is the $i$-th system pole corresponding to eigenvalue $\lambda_{i}$ of $\mathbf{A}$. Note that $c_{i}$ is the transfer function residue of the associated pole $p_{i}$. The transient response or ringdown of a SISO system is described by a sum of superimposed oscillations, given by (8) [1].

$$
y(t)=\sum_{i=1}^{n} \alpha_{i} e^{\sigma_{i} t} \cos \left(\omega_{i} t+\varphi_{i}\right)
$$

In discrete-time form, $y(t)$ is rewritten as:

$$
y[k]=y\left(k T_{s}\right)=\sum_{i=1}^{n} c_{i} z_{i}^{k}=\sum_{i=1}^{n} a_{i} e^{\sigma_{i} k T_{s}} \cdot \cos \left(\omega_{i} k T_{s}+\varphi_{i}\right), k=0,1, \ldots, N-1
$$

Ringdown analysis is loosely related to modal decomposition of the system impulse response. Specifically, the transfer function in FD is the Laplace transform of the system impulse response.

\subsubsection{Mode Identification Procedure}

To identify the modes contained in a ringdown response the generic procedure depicted in Figure 1 is used in SIEMToolbox. The mode estimates for each identification method target to minimize the mean absolute error (MAE) defined as:

$$
M A E=\frac{\sum_{k=0}^{N-1}\left|y[k]-\hat{y}\left[k \mid \theta_{M}\right]\right|}{N}
$$

where $\hat{y}\left[k \mid \boldsymbol{\theta}_{M}\right]$ is the ringdown response simulated by introducing in (10) the set of the estimated mode parameters $\boldsymbol{\theta}_{M}$. It should be noted, that dashed lines in Figure 1 refer to the optional procedures "Initialization" and "Optimal order determination", available in SIEMToolbox. More specifically, the initialization procedure is required for PEM [19] and hybrid FD/TD [24] methods, in order to determine an initial estimate of the mode parameters. Additionally, in order to develop models of the minimum order, i.e., described only by dominant modes, excluding any possible artificial modes, the optional "Optimal order determination" procedure is also available. The proposed procedure, depicted in Figure 1, identifies automatically the dominant modes contained in ringdown responses by the following steps:

- Step 1: The range of the examined model order is specified by the user; typically starting from 1 . Let us assume that in general form the lower model order value is $M_{\min }$ and the maximum $M_{\max }$.

- Step 2: The selected mode identification method is applied sequentially for each case in the range from $M_{\min }$ to $M_{\max }$; the ringdown response is simulated and the corresponding $M A E$ is calculated.

- Step 3: The calculated MAE of all examined $M_{\min }-M_{\max }$ cases are compared. The optimal model order corresponds to the case with the lower MAE. 


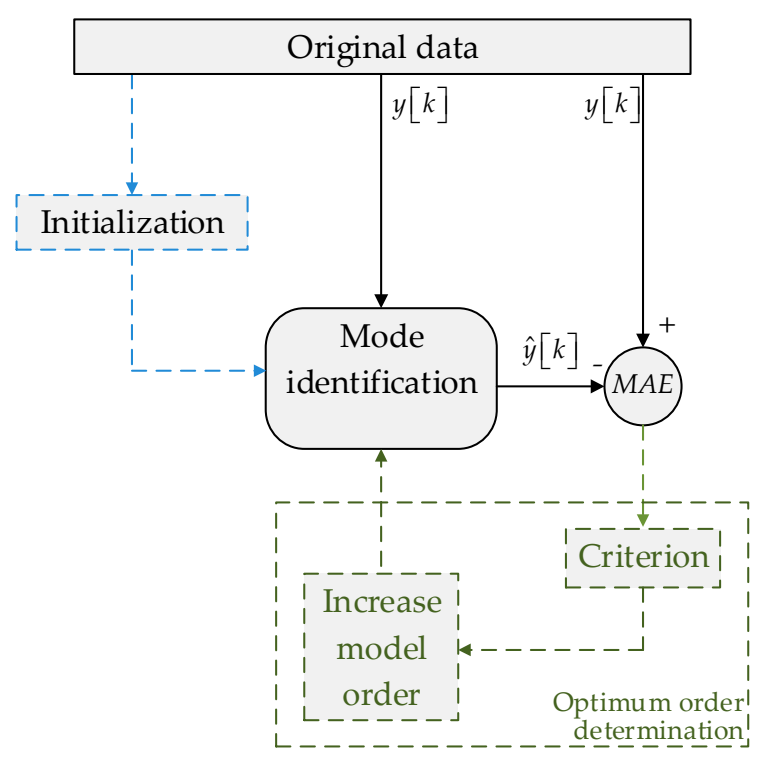

Figure 1. Mode identification procedure flowchart.

To further evaluate the accuracy of the estimated response the root mean square error (RMSE), the mean absolute percentage error (MAPE) and the coefficient of determination $\left(R^{2}\right)$ indexes defined in (11) and (13) are employed.

$$
\begin{gathered}
R M S E=\sqrt{\frac{\sum_{k=0}^{N-1}\left(y[k]-\hat{y}\left[k \mid \theta_{m}\right]\right)^{2}}{N}} \\
M A P E=\frac{100}{N} \cdot \sum_{k=0}^{N-1}\left|\frac{y[k]-\hat{y}\left[k \mid \theta_{m}\right]}{y[k]}\right| \\
R^{2}=\left(1-\frac{\sum_{k=0}^{N-1}\left(y[k]-\hat{y}\left[k \mid \theta_{m}\right]\right)^{2}}{\sum_{k=0}^{N}(y[k]-\bar{y})^{2}}\right) \cdot 100 \%
\end{gathered}
$$

Note that $\bar{y}$ is the mean value of the original ringdown signal.

\subsubsection{Multiple Signal Analysis}

To analyze multiple ringdown responses, the multi-signal approach of [37] is incorporated. Each identification method can be applied individually to each of the available $L$ signals and the corresponding modal estimates are calculated. The derived modal estimates are grouped based on the mode frequency. For each group of modes, a final value of the mode frequency $\frac{\bar{f}}{f_{i}}$ and damping factor $\overline{\sigma_{i}}$ is determined as the average value of the corresponding mode estimate as follows $[1,37]$ :

$$
\begin{gathered}
\overline{f_{i}}=\frac{\sum_{l=1}^{L} \hat{f}_{l}^{i}}{L} \\
\overline{\sigma_{i}}=\frac{\sum_{l=1}^{L} \hat{\sigma}_{l}^{i}}{L}
\end{gathered}
$$




\subsection{Network Equivalent Models}

Network equivalent models in general describe the real and reactive power response of a power system bus following a perturbation, e.g., change of bus voltage, short-circuit, etc. As already mentioned, static equivalent models express the real power $\left(P_{s}\right)$ and reactive power $\left(Q_{s}\right)$ at the bus as a function of the bus voltage $(V)$ and frequency $(f)$. The mathematical formulation of this dependence can be given in generalized form in (16) and (17), respectively, by means of functions $g_{s}$ and $h_{s}$ [28]:

$$
\begin{aligned}
& P_{s}=g_{s}\left(V, f \mid \theta_{P_{s}}\right) \\
& Q_{s}=h_{s}\left(V, f \mid \theta_{Q s}\right)
\end{aligned}
$$

where $\theta_{P s}$ and $\theta_{Q s}$ denote the set of parameters for the real and reactive power to be estimated, respectively.

Accordingly, the generalized formulation for the real power $\left(P_{d}\right)$ and reactive power $\left(Q_{d}\right)$ responses characterizing dynamic equivalent models is given in (18) and (19), respectively, including also the time dependence [28].

$$
\begin{aligned}
& P_{d}=g_{d}\left(V, f, t \mid \theta_{P d}\right) \\
& Q_{d}=h_{d}\left(V, f, t \mid \theta_{Q d}\right)
\end{aligned}
$$

Note that $\theta_{P d}$ and $\theta_{Q d}$ are the set of parameters for the real and reactive power, respectively, regarding dynamic models.

\subsubsection{Parameter Estimation Procedure}

The model parameters of (16), (17) and (18), (19) for each equivalent model structure can be estimated by means of non-linear least-squares (NLS) optimization. In NLS the response data are related to the simulated data by adjusting iteratively the model parameters to minimize the objective function $J$ given in (20), i.e., the error of the simulated response.

$$
J=\sum_{k=0}^{N-1}\left(y[k]-\hat{y}\left[k \mid \theta_{m}\right]\right)^{2}
$$

Here, $y[k]$ and $\hat{y}\left[k \mid \theta_{m}\right]$ are the original and the simulated data of the $k$-th sample, respectively and $\theta_{m}$ is the set of the model parameters, i.e., $\theta_{P s}, \theta_{Q s}, \theta_{P d}$ or $\theta_{Q d}$ at the $m$-th algorithm iteration. Note that, $y[k]$ can be $P_{s}, Q_{s}, P_{d}$ or $Q_{d}$. The flowchart of the SIEMToolbox parameter estimation procedure is presented in Figure 2. At each iteration $\hat{y}\left[k \mid \theta_{m}\right]$ is calculated, using the real or reactive power response expressions of the examined equivalent model as well as the corresponding set of parameters. Next, $J$ is evaluated, and the model parameters are updated since $J$ becomes less than a user-defined tolerance value $(\tau)$ or the maximum number of iterations $M$ is reached. In the case of network modelling by means of transfer function models, i.e., TF and TFNL models, in order to determine the optimal model order, the same iteration procedure, analyzed in Section 2.1.2., also applies.

\subsubsection{Equivalent Modelling with Multi-Signal Analysis}

Also, in the equivalent modelling feature, the model parameters can be estimated either using a single signal data set or multiple signals. If multiple signals are available, the parameters of the selected model are identified separately for each signal. The resulting model parameters using all signals are derived by averaging all relative estimated model parameter. 


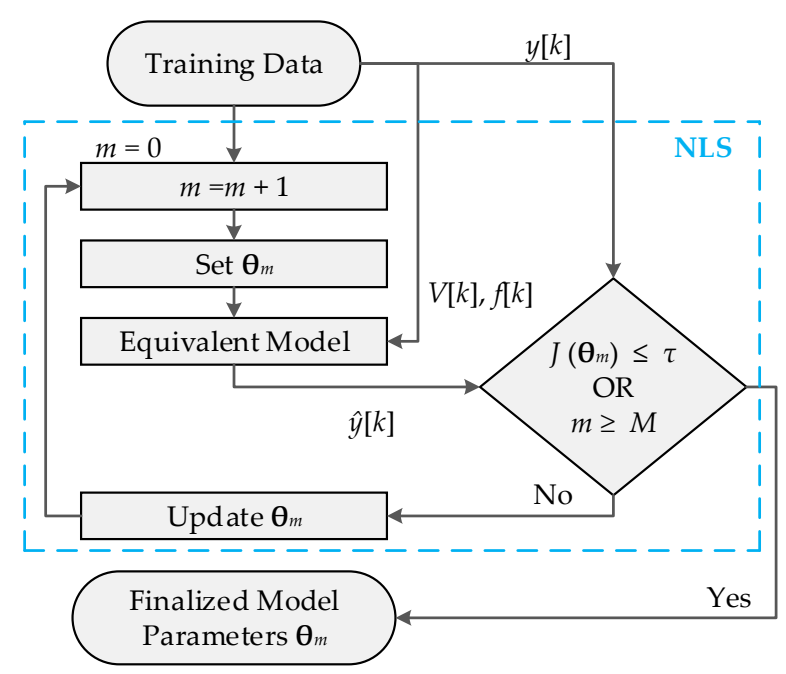

Figure 2. Flowchart of parameter estimation procedure.

\section{SIEMToolbox}

In the next sub-sections, the functionalities and the capabilities of the SIEMToolbox are presented in detail.

\subsection{Main Graphical User Interface of SIEMToolbox}

The SIEMToolbox is developed on the MATLAB programming language and can be launched by typing

$$
\text { >>main_gui }
$$

at the MATLAB prompt. The main window of the toolbox is depicted in Figure 3; from this window the mode identification and network equivalent modelling features can be selected.

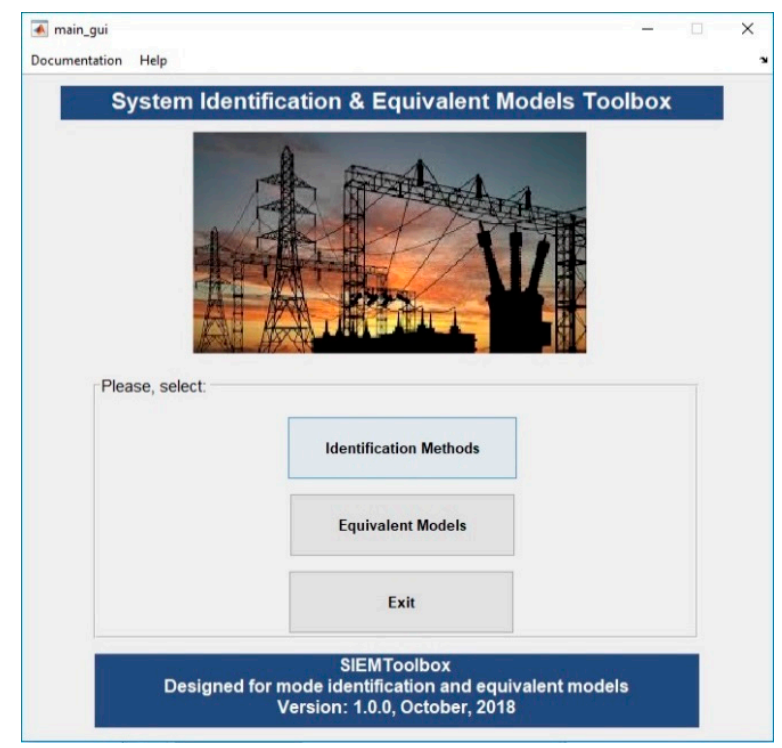

Figure 3. Main window of SIEMToolbox.

\subsection{Mode Identification Techniques}

In the mode identification techniques feature, the user must provide the measurement data, as shown in Figure 4. In addition to the selection of the type of the analysis, i.e., single- or multi-signal, the user must provide the input data in mat file extension, which must correspond to the following 
specifications and in an array form. Specifically, for single signal analysis, a $N \times 2$ array is required, where the first column corresponds to the observation time, whereas the second column to the signal response. For multiple signals, an $N \times W$ array is required, where $W-1$ is the number of signals. Accordingly, the first column contains the vector of the observation time. In each subsequent column, the $w$-th measured response data are stored.

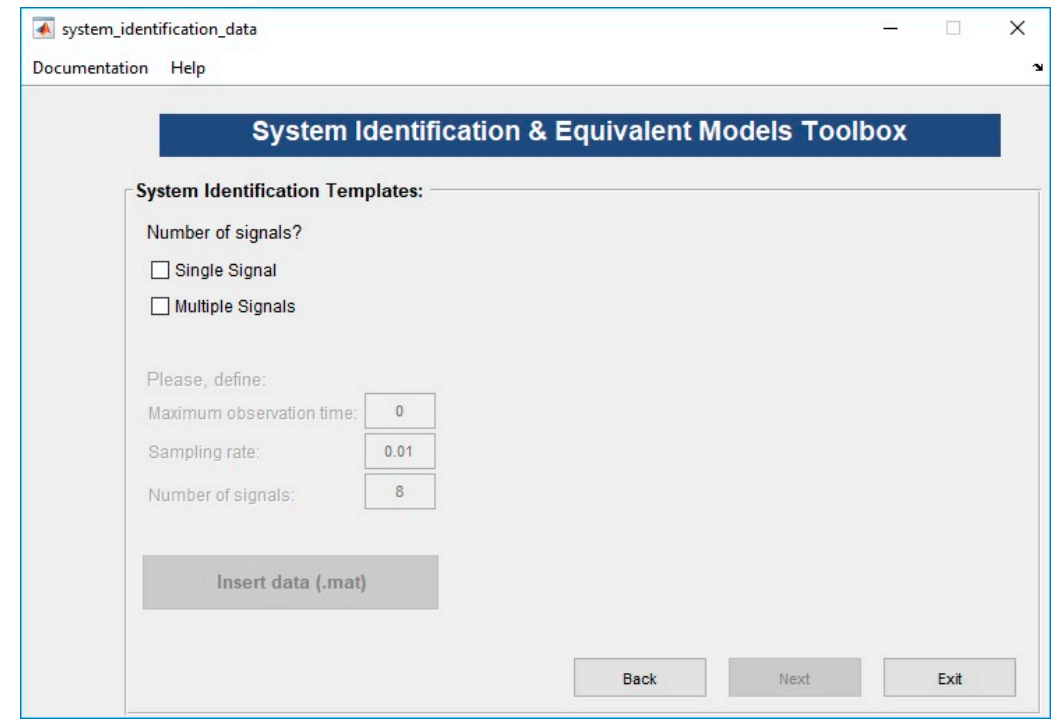

Figure 4. Mode identification techniques data input window.

After importing the measured data, the user can select one of the eight developed identification methods mentioned in Section 1.2. Consequently, the model order or a range for optimal order determination must be specified, as depicted in Figure 5.

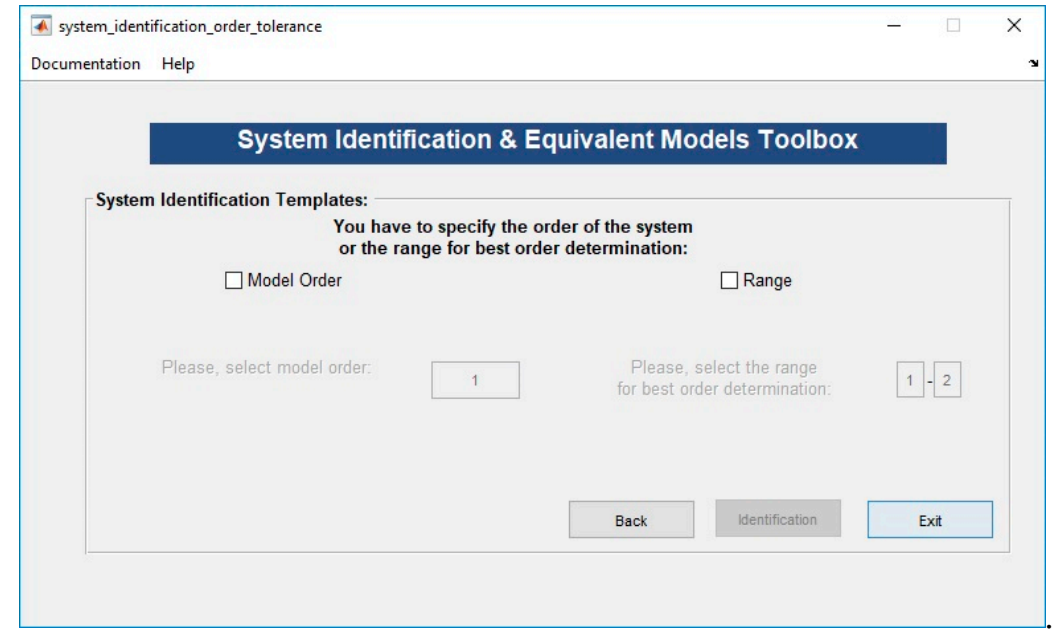

Figure 5. Model order determination window for mode identification method.

To visualize the findings of mode identification methods, the SIEMToolbox result window is employed. Indicative results are shown in Figure 6. Note that, in the example of Figure 6 the model order is selected equal to two, and single signal analysis is considered. 


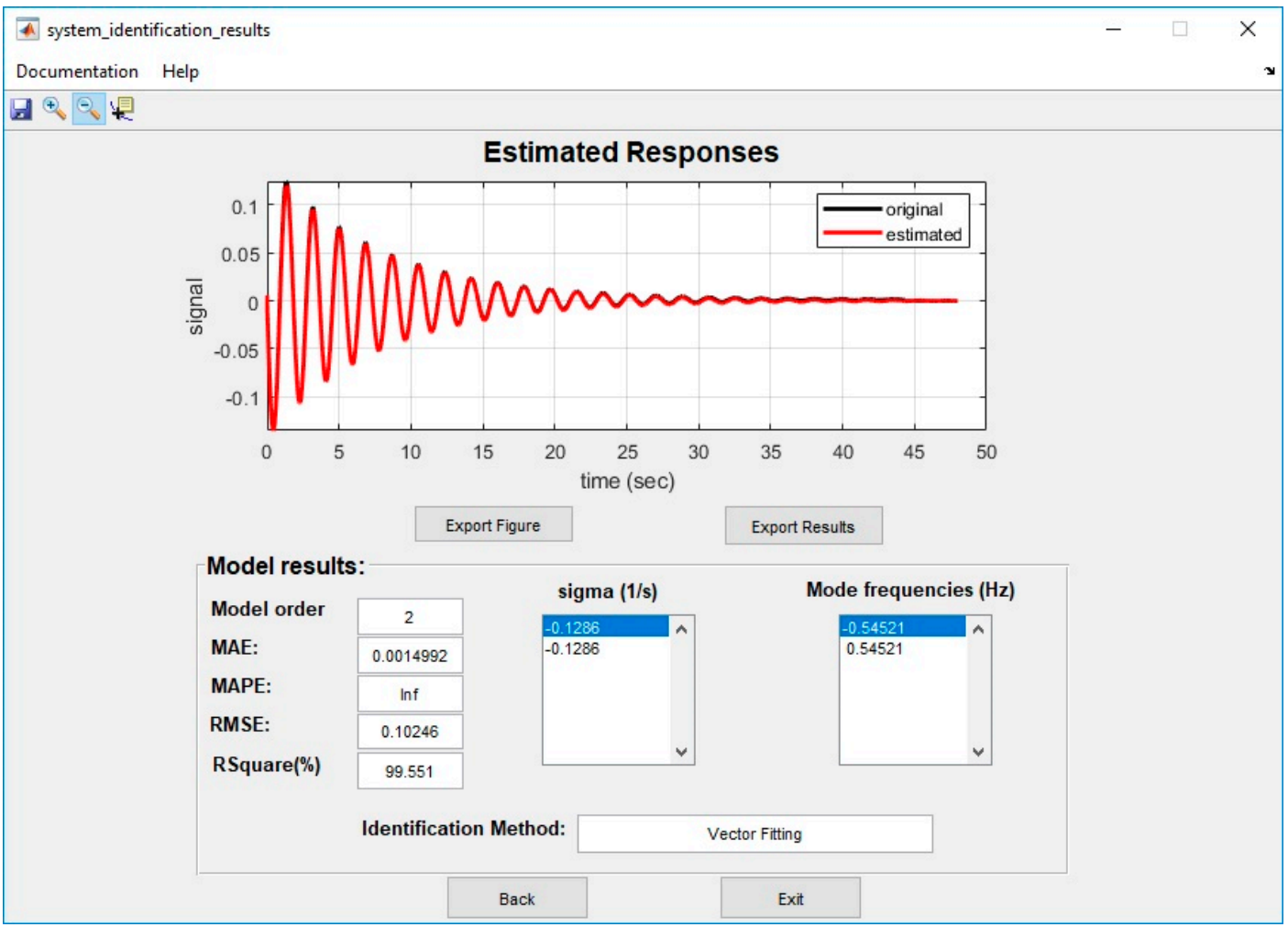

Figure 6. Mode identification result window. Indicative results for single signal analysis and predetermined model order.

Different capabilities are offered through the mode identification result window. For example, the measured (original) and the estimated response are compared, as depicted in the first frame of the window. Moreover, the calculated evaluation indexes, as well as the estimated damping factor and frequency of each mode is presented. Finally, it is worth mentioning that the user can export the plot or export the mode identification results in a txt file for further analysis.

\subsection{Network Equivalent Models}

Similar to mode identification techniques, a data input window is generated for the equivalent modelling option, as the one depicted in Figure 4. The equivalent model data input window comprises the same options as analyzed in Section 3.2. Also in this case, the input data must be provided in mat file extension. Five vectors namely $\mathbf{P}, \mathbf{Q}, \mathbf{f}, \mathbf{V}$ and $\mathbf{t}$, which correspond to the real power, reactive power, frequency, voltage and time response, respectively, are required. In the case of single signal analysis, each of the aforementioned responses must be stored in a different vector. Thus, in the mat file extension five $N \times 1$ vectors are included. In the case of multiple signals, the measurements must also be provided in five different vectors. However, in each column of the $\mathbf{P}, \mathbf{Q}, \mathbf{f}$ and $\mathbf{V}$ vectors, the $w$-th measured response is stored. Hence, one $N \times 1$ vector, i.e., $t$, and four $N \times W$ arrays are contained in the mat file, where $W$ is the number of signals. It should be emphasized that each column of the vectors $\mathbf{P}, \mathbf{Q}, \mathbf{f}$ and $\mathbf{V}$ should include the signal response for the exact time given from the time array, i.e., $t$.

Since the measurements are imported, the user can select the equivalent model under study described in Section 1.2. The corresponding window is shown in Figure 7. 


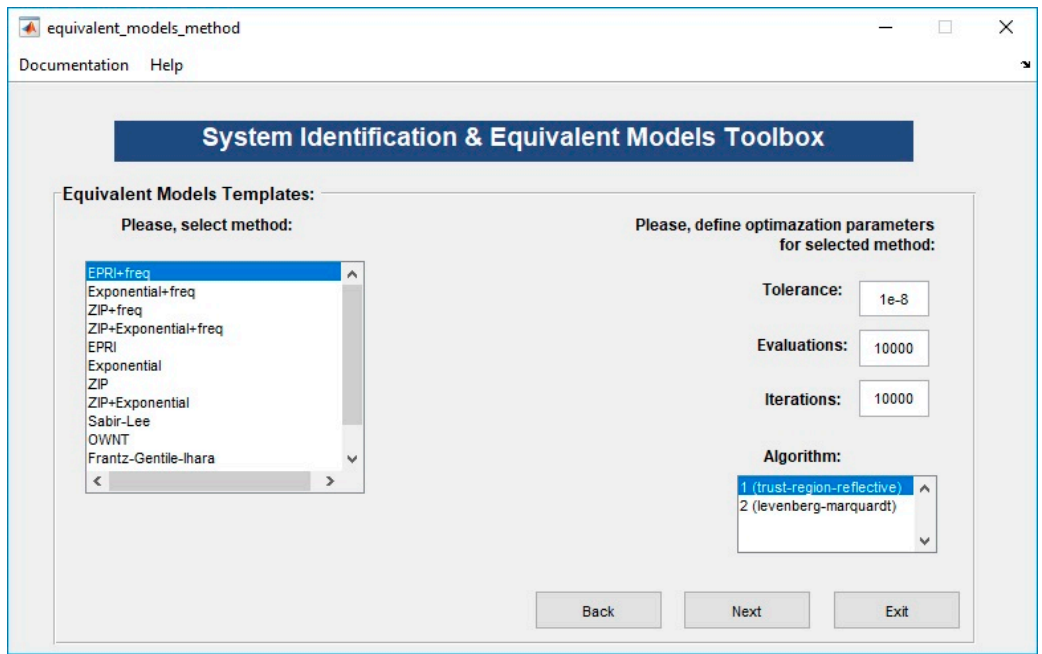

Figure 7. Equivalent method selection window.

Furthermore, the parameters for the NLS optimization must be defined. These parameters include the tolerance, the number of function evaluations and the number of total iterations. The default values are $10^{-8}, 10,000$ and 10,000, respectively. Two different algorithms are provided by the SIEMToolbox to solve the optimization problem, i.e., the trust-region-reflective and the Levenberg-Marquardt algorithm [38]. The next generated window differs depending on the selected analysis (single or multiple signal) as well as on the specified equivalent method. Subsequent to the selection of one of the models based on transfer functions, i.e., TF and TFNL, a similar window as the one depicted in Figure 5 is generated. Two different possibilities are offered. In the former, the user can choose the order of the transfer function for both real and reactive power, separately. In the latter, the SIEMToolbox automatically determines the best transfer function order within a range provided by the user, following the procedure described in Section 2.2.1.

The resulting window for the case of the equivalent models is visualized in Figure 8. Note that in this case, a model based on TF with a predetermined order and single signal input is selected.

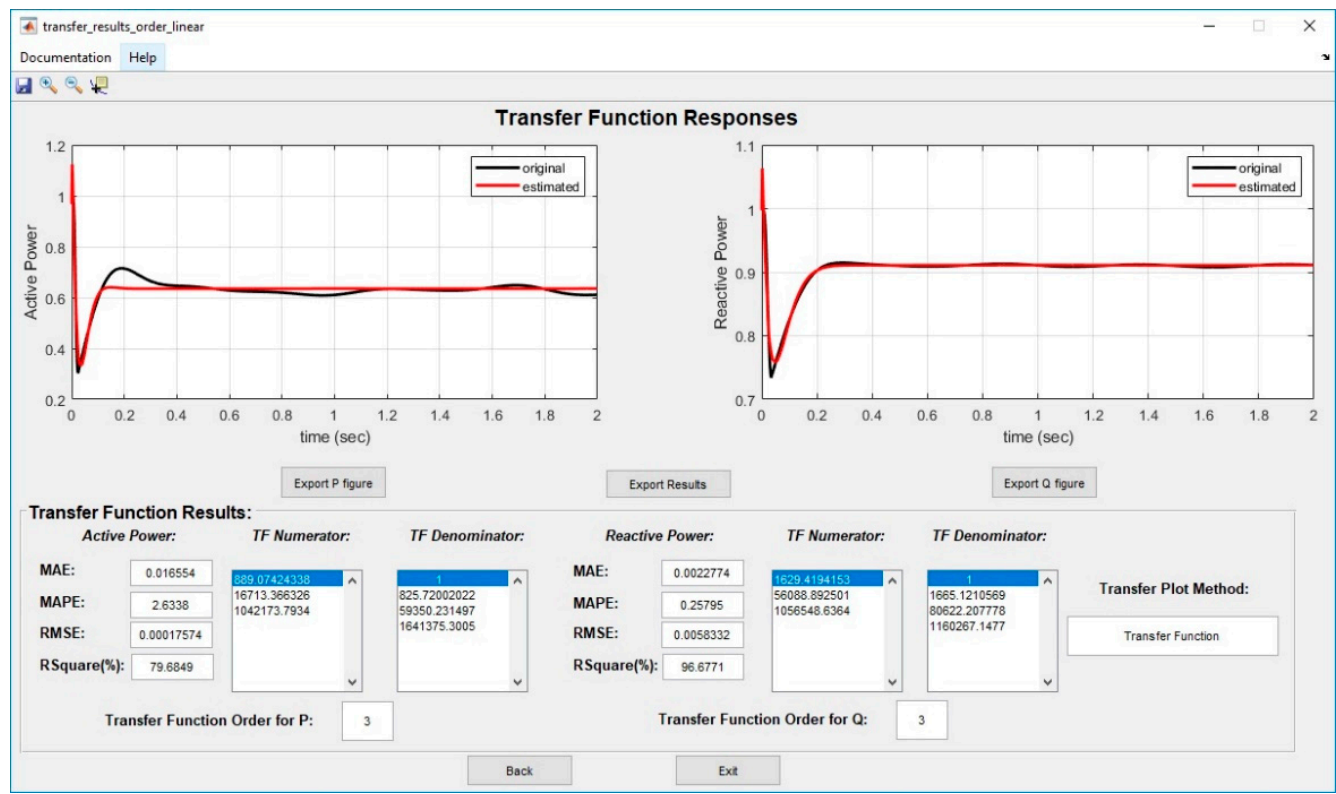

Figure 8. Equivalent model result window. Indicative results for unique input signal and specified transfer functions (TF) order. 
Similar to the mode identification result window multiple capabilities are offered. For example, the active and reactive power measured responses can be compared to the estimated, as depicted in Figure 8. In addition, the calculated evaluation indexes, as well as the calculated model parameters (or transfer function numerator and denominator results when transfer functions-based methods are considered) are presented. Finally, the user can export the real and reactive power plots for further processing or export the results in a txt file.

\section{Results}

In this section, the effectiveness of the developed SIEMToolbox is evaluated. Indicative results of mode identification and network equivalencing are presented.

\subsection{Description of the Systems under Study}

The performance of the mode identification and equivalent modeling techniques is assessed using signal responses obtained from the Kundur two-area system topology [36] and a laboratory-scale ADN [39], respectively. In the following Sections the network topologies employed to obtain the dynamic responses are briefly described.

\subsubsection{Kundur's Two-Area System}

Kundur's two-area system consists of eleven buses and two areas, connected by a weak tie between bus 7 and 9. The basic topology is depicted in Figure 9. In total, two loads are applied to the system at bus 7 and 9. Two shunt capacitors are also connected to bus 7 and 9. More information regarding the network can be found in [36].

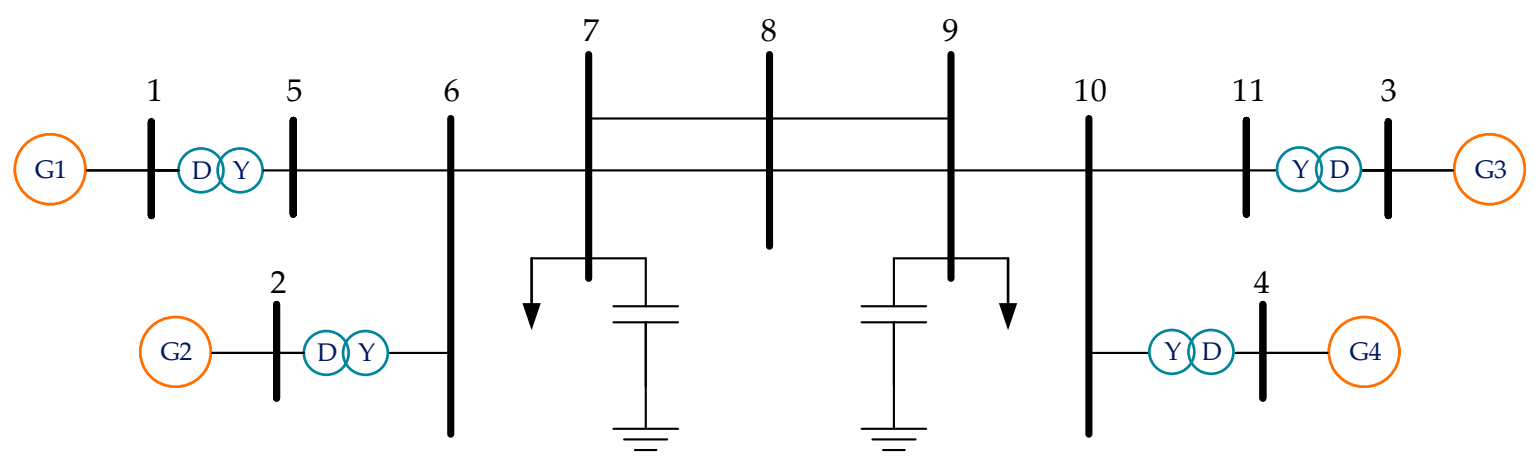

Figure 9. Kundur's two-area system topology.

In order to obtain the measurements for the assessment of the mode identification methods, a disturbance at bus 7 is caused. Specifically, a load with nominal power equal to $350 \mathrm{MW}$ is disconnected from the bus at the time instant $t=1 \mathrm{~s}$ and is re-connected at $t=1.2 \mathrm{~s}$.

\subsubsection{Laboratory-Scale Active Distribution Network}

To evaluate the performance of the developed equivalent models, a series of experiments were conducted, using the three-phase, $400 \mathrm{~V}, 50 \mathrm{~Hz}$, laboratory-scale ADN of Figure 10. Two sub-grids conclude the test setup. The examined topology is interconnected with the main utility grid, which is simulated using a real-time digital simulator (RTDS) and is connected to the ADN by means of a three-phase programmable voltage source (PVS). A detailed description regarding the examined topology can be found in [39]. 


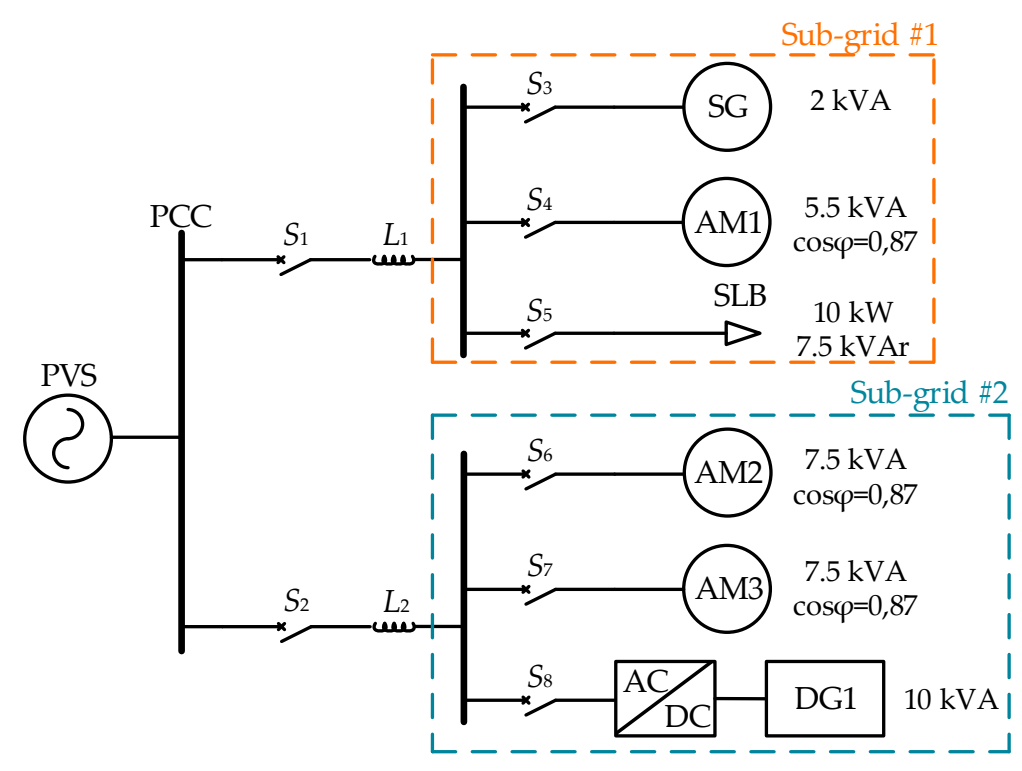

Figure 10. Laboratory-scale active distribution network (ADN).

Different network configurations were investigated by connecting/disconnecting the switches of the test setup $\left(S_{1}-S_{8}\right)$. In addition, different load operating conditions were examined by varying the power of the installed components. Dynamic responses of real and reactive power were acquired by causing artificial voltage disturbances using the PVS, ranging between -0.1 p.u. and 0.1 p.u. To verify the efficiency of the network equivalent models developed within SIEMToolbox the following topologies were examined:

- Topology 1: The static load bank and the asynchronous machine (AM1) are connected to the network. The AM1 operates as a motor.

- Topology 2: The static load bank, the inverter interfaced distributed generator (DG1), the synchronous generator (SG) and all the asynchronous machines are connected to the network. In this case, the AM2 and AM3 asynchronous machines operate as motors, whereas the AM1 operates as generator.

\subsection{Mode Identification}

The effectiveness of SIEMToolbox to analyze ringdown responses is evaluated in the following subsections. For this purpose, indicative results for single- and multi-signal analysis are presented.

\subsubsection{Single Signal Analysis}

To evaluate the performance of the mode identification techniques the real power response of Bus 7 acquired with a sampling rate of 100 samples per second is used. The recorded ringdown response is first detrended to remove possible DC components and then the transient part of the response is neglected and only the oscillatory part is considered to focus on the analysis of the interarea mode.

The influence of the mode identification model order is demonstrated in Figure 11, where the original real power response acquired from NEPLAN software is compared with the corresponding responses simulated by the Prony method assuming varying model order. As shown in Figure 11, a high model order is required for the Prony method to accurately capture the original real power response. 


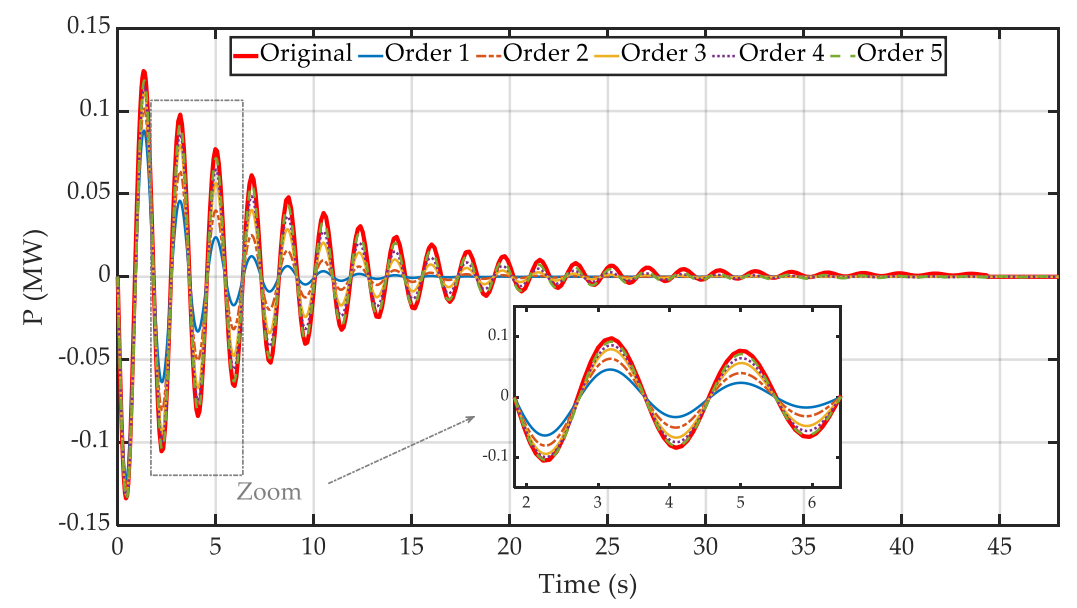

Figure 11. Impact of model order. Prony method.

The influence of the model order is further assessed by comparing the corresponding identified modes with the true interarea mode calculated by the NEPLAN eigenvalue analysis tool [40], i.e., damping factor and frequency equal to $-0.127 \mathrm{~s}^{-1}$ and $0.54 \mathrm{~Hz}$, respectively. The resulting identified modes are depicted in Figure 12 for varying model order. As shown in the magnified area of Figure 12, by increasing the model order more accurate approximations are provided for the estimation of the interarea mode. Furthermore, it is interesting to mention that as the model order increases, the estimated modal frequency remains constant, while the damping factor changes. It should be also noted that, all remaining modes are trivial artificial modes introduced to enhance the accuracy of the method $[25,41]$.

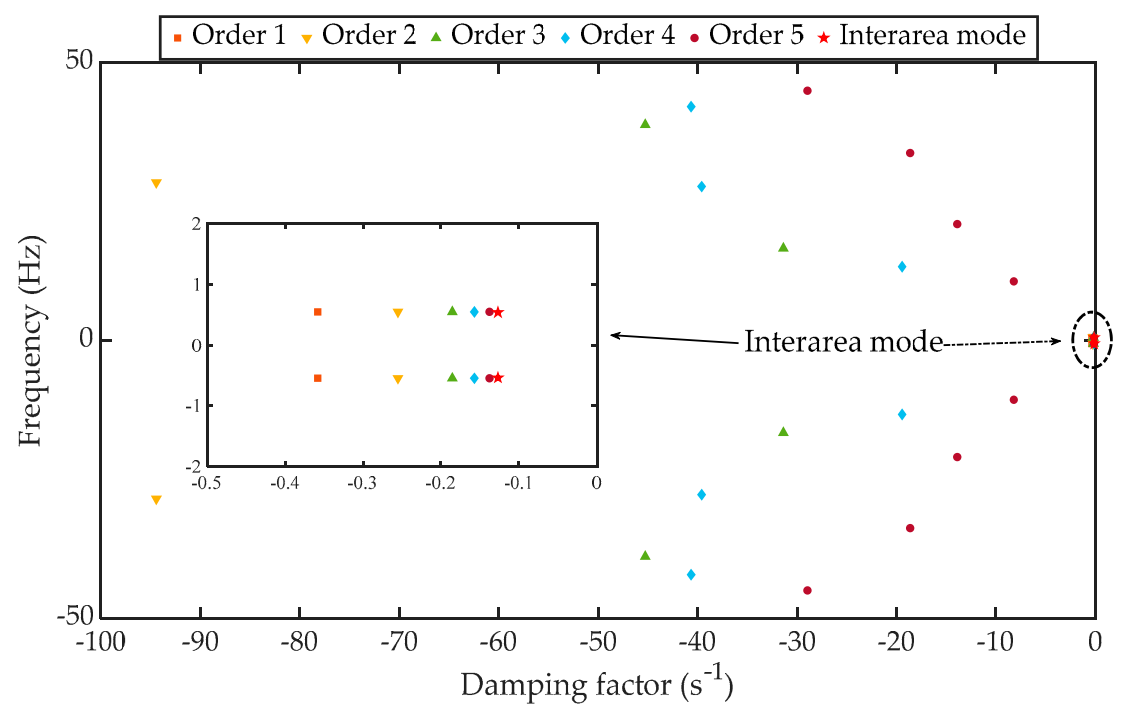

Figure 12. Eigenvalue analysis for Prony method assuming different model orders.

\subsubsection{Multiple Signal Analysis}

To offer a further insight on the accuracy of the identification methods, the multi-signal approach is employed in this subsection. The examined mode identification techniques are applied individually to eight distinct signals and the corresponding modal parameters are estimated. The resulting averaged interarea modes are compared with the true NEPLAN interarea mode $\left(\sigma=-0.127 \mathrm{~s}^{-1}, f=0.54 \mathrm{~Hz}\right)$. For this purpose, the median absolute deviation $(M A D)$ is employed [25], which is defined as:

$$
M A D=\operatorname{median}(|\hat{\lambda}-\lambda|)
$$


where $\hat{\lambda}$ denotes the modal estimate and $\lambda$ is the true interarea mode. Note that the comparison is carried out assuming first order approximations for all methods. The associated $M A D$ results of the interarea mode using all available methods in the SIEMToolbox are compared in Table 1. It should be clarified that the resulting damping factor and frequency for each method is the averaged value by means of multiple signals.

Table 1. Impact of multi-signal analysis on the accuracy of the mode estimates. Mean values acquired from eight signals.

\begin{tabular}{cccc}
\hline Technique & Damping Factor $\left(\mathbf{s}^{\mathbf{- 1}}\right)$ & Frequency $\mathbf{( H z )}$ & MAD \\
\hline MP & -0.12869 & 0.54086 & 0.0009 \\
Prony & -0.40470 & 0.56803 & 0.1396 \\
ERA & -0.12848 & 0.54143 & 0.0010 \\
N4SID & -0.61850 & 0.74605 & 0.2665 \\
PEM & -0.24762 & 0.67708 & 0.0913 \\
FFT & -0.13493 & 0.54155 & 0.0040 \\
VF & -0.13062 & 0.54371 & 0.0026 \\
Hybrid FD/TD & -0.13228 & 0.54404 & 0.0033 \\
\hline
\end{tabular}

Based on the results of Table 1, it is evident that MP, ERA, VF and Hybrid FD/TD methods present higher accuracy regarding the interarea mode estimates, compared with the rest of the identification methods.

\subsection{Equivalent Modelling}

In this subsection, the SIEMToolbox parameter estimation procedure is applied to evaluate the performance of the network equivalent models by means of the simulated dynamic real and reactive power responses.

\subsubsection{Single Signal Analysis}

One instant of the available real and reactive power responses of the laboratory-scale ADN Topology 2 is used to evaluate the performance of the developed equivalent models. Initially, the effect of the model order of TFNL is investigated. As demonstrated in Table 2, the accuracy of the simulated real and reactive power increases with the model order. Specifically, optimal results are obtained using model order 5 and 4 for the real power and the reactive power, respectively.

Table 2. Mean absolute error (MAE) for the modelling of a real/reactive power response of Topology 2, using transfer functions and non-linear equations (TFNL) model.

\begin{tabular}{ccc}
\hline Model Order & Real Power & Reactive Power \\
\hline 1 & 0.0216 & 0.0042 \\
2 & 0.0380 & 0.0026 \\
3 & 0.0238 & 0.1262 \\
4 & 0.0315 & 0.0014 \\
5 & 0.0103 & 0.4876 \\
\hline
\end{tabular}

Additionally, to provide a better insight, the modelling of the specific real power response assuming selective model orders is illustrated in Figure 13. It should be noted that in all cases, each power response is normalized by means of the pre-disturbance value. In Figure 13 is verified that higher model order results in more accurate simulations; this is mostly important in cases where the dynamic load penetration in the whole load mix is large, since the oscillatory behavior in such cases is more complex. 


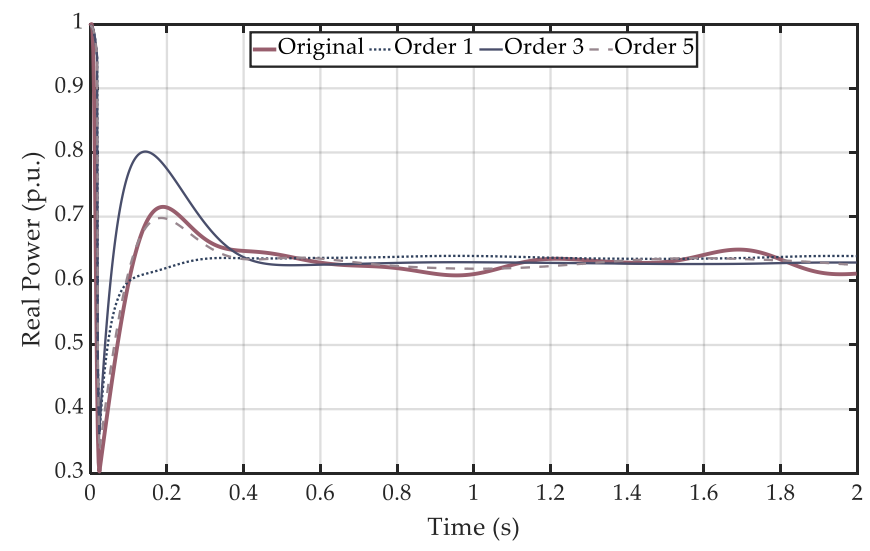

Figure 13. Modelling of real power. Estimations provided by the TFNL using different model orders.

Next, to provide a thorough insight in the accuracy of the network equivalent models, in Figure 14, the real and reactive power responses are simulated using some of the most widely used static equivalents as well as the two dynamic equivalent models of SIEMToolbox. Note that for the dynamic network equivalent models optimal order is considered; for the modelling of the reactive power, model order 4 is considered for both dynamic models, whereas model order 4 and 5 is assumed for the modelling of the real power for the TF and TFNL models, respectively.
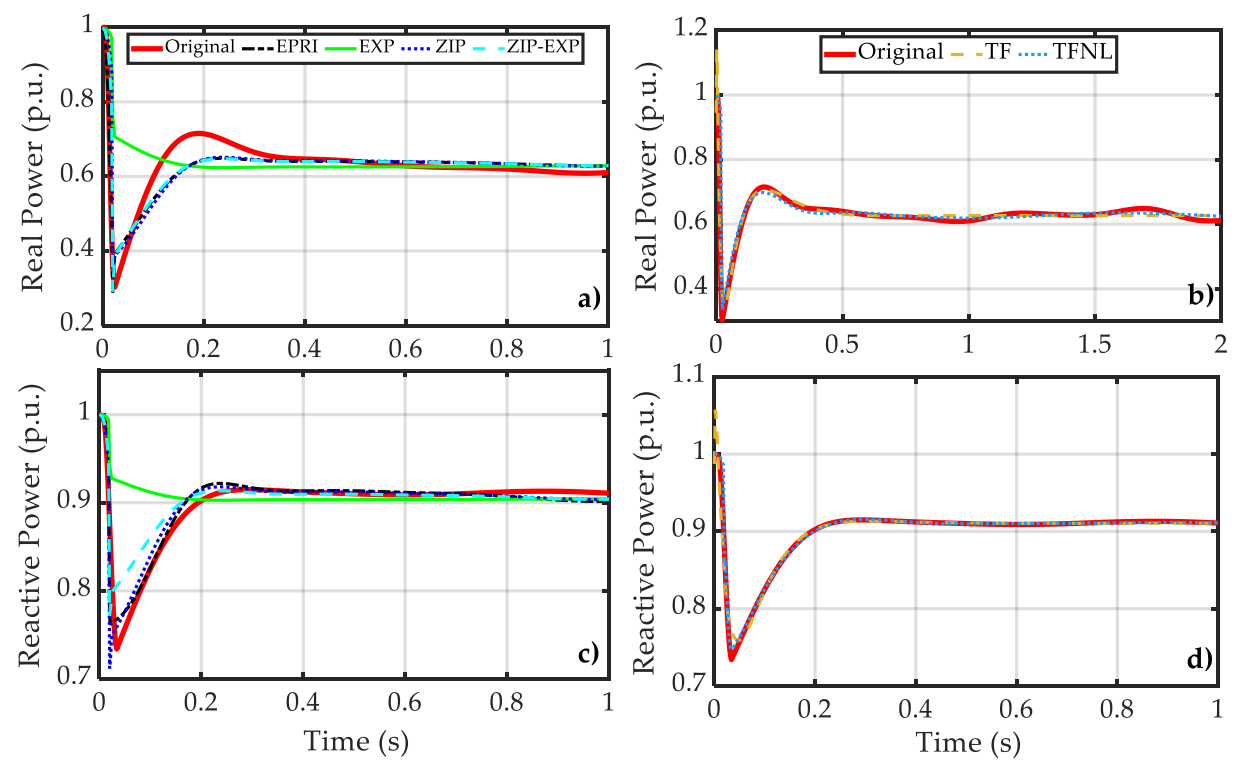

Figure 14. Modelling of (a) real and (b) reactive power response using static models; Modelling of (c) real and (d) reactive power response using dynamic equivalent models.

To evaluate the accuracy of the derived network equivalent models, the following evaluation indexes are also employed [35]:

$$
\begin{array}{r}
\operatorname{OE}(\%)=\left|\frac{y_{+}-\hat{y}_{+}}{y_{+}}\right| \cdot 100 \% \\
\operatorname{SSE}(\%)=\left|\frac{y_{s s}-\hat{y}_{s s}}{y_{s s}}\right| \cdot 100 \%
\end{array}
$$

The overshoot error, i.e., $O E(\%)$ in (22), denotes the percentage relative error for the estimation of the power overshoot, whereas the steady-state error, i.e., SSE (\%) in (23), is the percentage relative error related with the approximation of the new steady-state. Note that $y_{+}$is the measured power 
immediately after the voltage sag, while $y_{s s}$ is the power at the new steady-state condition. Finally, $\hat{y}_{+}$ and $\hat{y}_{s s}$ denote the corresponding estimations provided by the examined equivalent model.

In Figure 15, the $O E$ and SSE values for each model are depicted for the real and the reactive power. It is shown that two dynamic equivalent models result into lower OE (\%) and SSE (\%) compared to the static models.
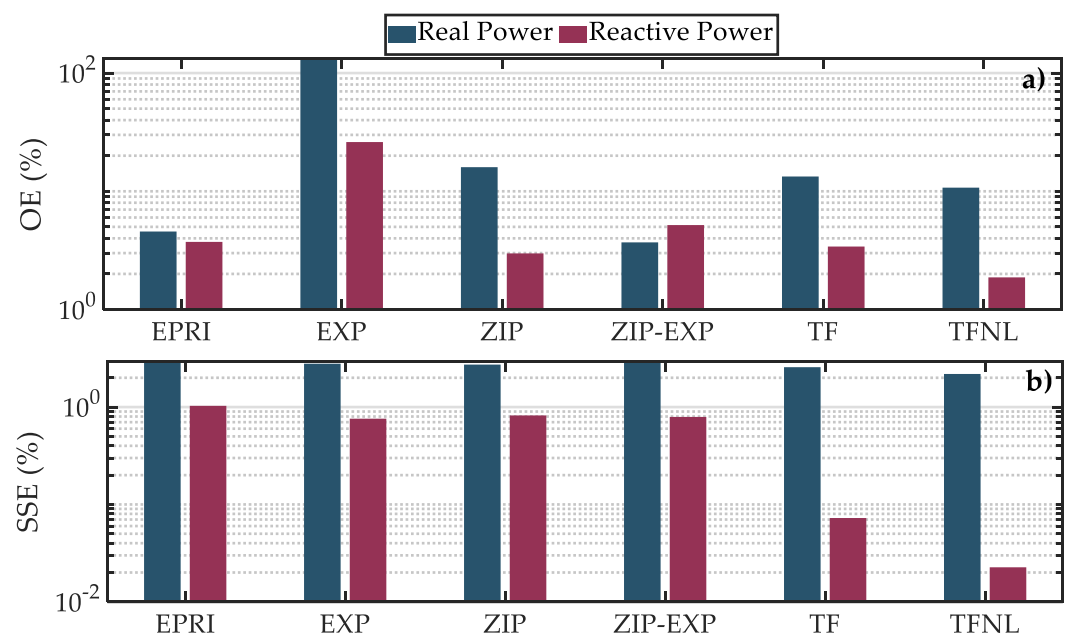

Figure 15. (a) Overshoot and (b) steady-state percentage relative errors. Note that the results are illustrated using a logarithmic scale.

\subsubsection{Multiple Signal Analysis}

In this subsection the performance of all available network equivalent models supported by SIEMToolbox are further evaluated using multiple signal responses. Specifically, in Figure 16 the resulting mean values of MAPE, over 10 responses of Topology 1, are depicted for both the real and reactive power. The presented values verify that dynamic models based on transfer functions, i.e., TF and TFNL, present higher accuracy compared to the static equivalents. Note that for the TF and the TFNL equivalent models optimal order determination was considered. Furthermore, it should be mentioned that regarding the static equivalent models, the dynamic behavior of the ADN is simulated more accurately by using the ZIP model. Another interesting remark is that the static equivalent models that consider frequency dependency, i.e., EPRI- $f$, EXP-f, ZIP- $f$ and ZIP-EXP-f, do not improve significantly the accuracy of the simulated responses.
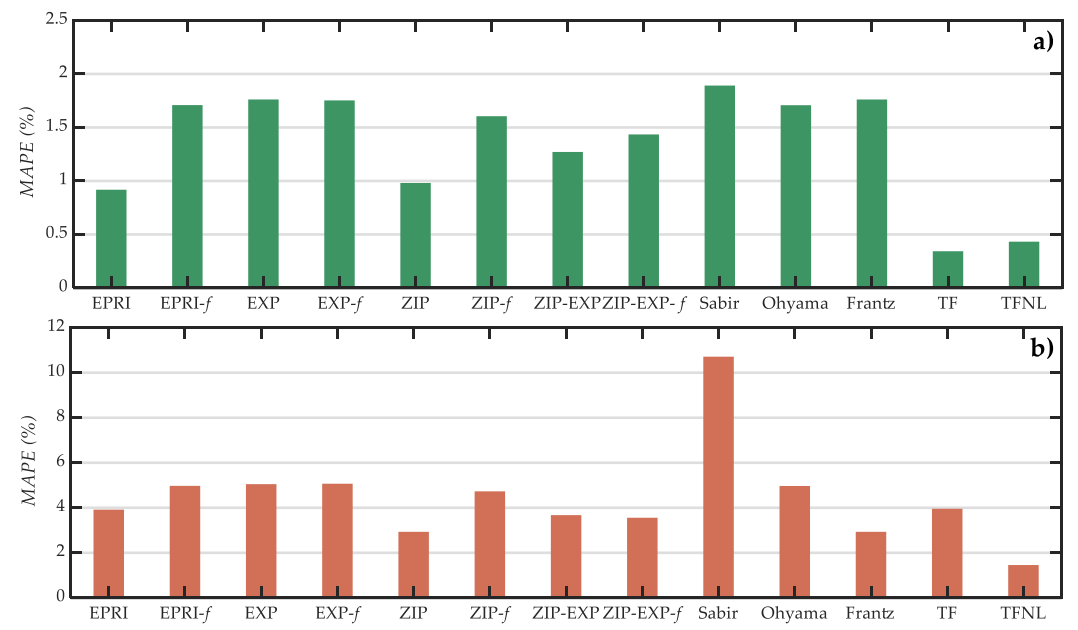

Figure 16. Average of mean absolute percentage error (MAPE) index using multiple responses; (a) real and $(\mathbf{b})$ reactive power. 


\section{Conclusions}

In this paper, the SIEMToolbox, which was developed in MATLAB environment and is provided with a complete user-friendly GUI, is demonstrated. SIEMToolbox incorporates several measurement-based methods for the analysis and testing of mode identification techniques and network equivalent models. The theoretical background regarding mode identification and network equivalencing by using dynamic responses is analyzed. The mode identification and parameter estimation procedures implemented within SIEMToolbox are described thoroughly and the corresponding basic features and settings of SIEMToolbox are presented to demonstrate the applications for the derivation of measurement-based equivalent models.

Indicative results using single- and multi-signal analysis are employed to assess the performance of the toolbox in analyzing ringdown responses. For this purpose, simulated dynamic responses from the Kundur two-area system are employed. Finally, the SIEMToolbox parameter estimation procedure is applied to various static and dynamic model structures to evaluate their performance in modelling real and reactive power dynamic responses of $\mathrm{ADNs}$, using real measurements acquired from a laboratory-scale ADN.

Author Contributions: Writing, conceptualization and modelling, E.O.K.; writing—original draft preparation and investigations, G.A.B.-N.; software implementation and formal analysis, K.A.S.; writing-review and editing, T.A.P.; supervision, G.K.P.

Funding: This research received no external funding.

Conflicts of Interest: The authors declare no conflict of interest.

\section{References}

1. Task Force on Identification of Electromechanical Modes. Identification of Electromechanical Modes in Power Systems; Technical Report PES-TR15; IEEE Power \& Energy Society: Piscataway, NJ, USA, 2012; Available online: http://sites.ieee.org/pes-resource-center/files/2013/11/TR15_Modal_Ident_TF_Report.pdf (accessed on 7 June 2019).

2. Ma, F.; Vittal, V. Right-Sized Power System Dynamic Equivalents for Power System Operation. IEEE Trans. Power Syst. 2011, 26, 1998-2005. [CrossRef]

3. Krommydas, K.F.; Alexandridis, A.T. Modular Control Design and Stability Analysis of Isolated PV-Source/Battery-Storage Distributed Generation Systems. IEEE Trans. Emerg. Sel. Top. Circuits Syst. 2015, 5, 372-382. [CrossRef]

4. Feng, X.; Lubosny, Z.; Bialek, J. Dynamic equivalencing of distribution network with high penetration of distributed generation. In Proceedings of the 41st International Universities Power Engineering Conference, Newcastle-upon-Tyne, UK, 6-8 September 2006; pp. 467-471.

5. Ishchenko, A.; Myrzik, J.; Kling, W. Dynamic equivalencing of distribution networks with dispersed generation using Hankel norm approximation. IET Gener. Transm. Distrib. 2007, 1, 818-825. [CrossRef]

6. Arif, A.; Wang, Z.; Wang, J.; Mather, B.; Bashualdo, H.; Zhao, D. Load modeling-a review. IEEE Trans. Smart Grid 2018, 9, 5986-5999. [CrossRef]

7. Thambirajah, J.; Barocio, E.; Thornhill, N. Comparative review of methods for stability monitoring in electrical power systems and vibrating structures. IET Gener. Transm. Distrib. 2010, 4, 1086-1103. [CrossRef]

8. Sun, K.; Zhou, Q.; Liu, Y. A phase locked loop-based approach to real-time modal analysis on synchrophasor measurements. IEEE Trans. Smart Grid 2014, 5, 260-269. [CrossRef]

9. Trudnowski, D.J.; Pierre, J.W. Overview of algorithms for estimating swing modes from measured responses. In Proceedings of the IEEE Power and Energy Society General Meeting, Calgary, AB, Canada, 26-30 July 2009.

10. Chaudhuri, N.R.; Domahidi, A.; Majumder, R.; Chaudhuri, B.; Korba, P.; Ray, S.; Uhlen, K. Wide-area power oscillation damping control in nordic equivalent system. IET Gener. Transm. Distrib. 2010, 4, 1139-1150. [CrossRef]

11. Messina, A.R. Inter-area Oscillations in Power Systems: A Nonlinear and Nonstationary Perspective; Springer: Berlin, Germany, 2009; ISBN 978-0-387-89529-1. 
12. Kontis, E.O.; Papadopoulos, T.A.; Chrysochos, A.I.; Papagiannis, G.K. Measurement-Based Dynamic Load Modeling Using the Vector Fitting Technique. IEEE Trans. Power Syst. 2018, 33, 338-351. [CrossRef]

13. Golpîra, H.; Seifi, H.; Haghifam, M.R. Dynamic equivalencing of an active distribution network for large-scale power system frequency stability studies. IET Gener. Transm. Distrib. 2015, 9, 2245-2254. [CrossRef]

14. Robitzky, L.; Mayorga-Gonzalez, D.; Kittl, C.; Strunck, C.; Zwartscholten, J.; Muller, S.C.; Hager, U.; Myrzik, J.; Rehtanz, C. Impact of active distribution networks on voltage stability of electric power systems. In Proceedings of the IREP Bulk Power Systems Dynamics and Control Symposium, Espinho, Portugal, 1 August 2017.

15. SIEMToolbox. Available online: http://power.ee.auth.gr/ (accessed on 7 June 2019).

16. Hauer, J.F.; Demeure, C.J.; Scharf, L.L. Initial results in Prony analysis of power system response signals. IEEE Trans. Power Syst. 1990, 5, 80-89. [CrossRef]

17. Crow, M.L.; Singh, A. The matrix pencil for power system modal extraction. IEEE Trans. Power Syst. 2005, 20, 501-502. [CrossRef]

18. Sanchez-Gasca, J.J.; Chow, J.H. Performance comparison of three identification methods for the analysis of electromechanical oscillations. IEEE Trans. Power Syst. 1999, 14, 995-1002. [CrossRef]

19. Ljung, L. System Identification: Theory for the User; Prentice-Hall PTR: Upper Saddle River, NJ, USA, 1999.

20. Katayama, T. Subspace Methods for System Identification; Springer: Berlin, Germany, 2005; ISBN 978-1-85233-981-4.

21. O'Shea, P. The use of sliding spectral windows for parameter estimation in power system disturbance monitoring. IEEE Trans. Power Syst. 2000, 15, 1261-1267. [CrossRef]

22. Gustavsen, B.; Semlyen, A. Rational approximation of frequency domain responses by vector fitting. IEEE Trans. Power Deliv. 1999, 14, 1261-1267. [CrossRef]

23. Papadopoulos, T.A.; Chrysochos, A.I.; Kontis, E.O.; Papagiannis, G.K. Ringdown analysis of power systems using vector fitting. Electr. Power Syst. Res. 2016, 141, 100-103. [CrossRef]

24. Papadopoulos, T.A.; Chrysochos, A.I.; Kontis, E.O.; Papadopoulos, P.N.; Papagiannis, G.K. Measurement-Based Hybrid Approach for Ringdown Analysis of Power Systems. IEEE Trans. Power Syst. 2016, 31, 4435-4446. [CrossRef]

25. Kontis, E.O.; Papadopoulos, T.A.; Barzegkar-Ntovom, G.A.; Chrysochos, A.I.; Papagiannis, G.K. Modal analysis of active distribution networks using system identification techniques. Int. J. Electr. Power Energy Syst. 2018, 100, 365-378. [CrossRef]

26. Task Force on Load Representation for Dynamic Performance. Load representation for dynamic performance analysis (of power systems). IEEE Trans. Power Syst. 1993, 8, 472-482. [CrossRef]

27. Task Force on Load Representation for Dynamic Performance. Bibliography on load models for power flow and dynamic performance simulation. IEEE Trans. Power Syst. 1995, 10, 523-538. [CrossRef]

28. CIGRE WG C4.605 Report. Modelling and Aggregation of Loads in Flexible Power Networks; CIGRE: Paris, France, 2013.

29. EPRI. Extended Transient-Midterm Stability Program Package FACTS Version User's Manual, EPRI Project 1208-9; EPRI: Palo Alto, CA, USA, 1990.

30. Li, Y.; Chiang, H.D.; Choi, B.K.; Chen, Y.T.; Huang, D.H.; Lauby, M.G. Representative static load models for transient stability analysis: Development and examination. IET Gener. Transm. Distrib. 2007, 1, 422-431. [CrossRef]

31. Sabir, S.A.Y; Lee, D.C. Dynamic Load Models Derived from Data acquired during System Transients. IEEE Trans. Power Appar. Syst. 1982, PAS-I01, 3365-3372. [CrossRef]

32. Ohyama, T.; Walanabe, A.; Nishimura, K.; Tsuruta, S. Voltage Dependence on Composite Loads in Power Systems. IEEE Trans. Power Appar. Syst. 1985, PAS-104, 3064-3073. [CrossRef]

33. Frantz, T.; Gentile, T.; Ihara, S.; Simons, N.; WaIdron, M. Load Behavior Observed in LILCO and RG\&E Systems. IEEE Trans. Power Appar. Syst. 1984, PAS-103, 819-831.

34. Lin, C.J.; Chen, A.Y.T.; Chiou, C.Y.; Huang, C.H.; Chiang, H.D.; Wang, J.C.; Fekih-Ahmed, L. Dynamic load models in power systems using the measurement approach. IEEE Trans. Power Syst. 1993, 8, 309-315. [CrossRef]

35. Kontis, E.O.; Dimitrakopoulos, S.P.; Chrysochos, A.I.; Papagiannis, G.K.; Papadopoulos, T.A. Dynamic equivalencing of active distribution grids. In Proceedings of the IEEE Manchester Power Tech, Manchester, UK, 18-22 June 2017.

36. Kundur, P. Power System Stability and Control; McGraw-Hill: New York, NY, USA, 1994. 
37. Ning, J.; Sarmadi, S.A.N.; Venkatasubramanian, V. Two-level ambient oscillation modal estimation from synchrophasor measurements. IEEE Trans. Power Syst. 2015, 30, 2913-2922. [CrossRef]

38. Ljung, L. System Identification Toolbox: User's Guide; The Mathworks Inc.: Natick, MA, USA, 2013.

39. Kontis, E.O.; Papadopoulos, T.A.; Syed, M.H.; Guillo-Sansano, E.; Burt, G.M.; Papagiannis, G.K. Artificial-intelligence method for the derivation of generic aggregated dynamic equivalent models. IEEE Trans. Power Syst. 2019, 34, 2947-2956. [CrossRef]

40. NEPLAN Help; NEPLAN Version 5.5.5 R1; NEPLAN: Küsnacht, Switzerland, 1988-2010.

41. Zhou, N.; Pierre, J.W.; Trudnowski, D. A stepwise regression method for estimating dominant electromechanical modes. IEEE Trans. Power Syst. 2012, 27, 1051-1059. [CrossRef]

(C) 2019 by the authors. Licensee MDPI, Basel, Switzerland. This article is an open access article distributed under the terms and conditions of the Creative Commons Attribution (CC BY) license (http://creativecommons.org/licenses/by/4.0/). 